\section{BMJ Open} Ophthalmology

\title{
Current and upcoming anti-VEGF therapies and dosing strategies for the treatment of neovascular AMD: a comparative review
}

\author{
Saira Khanna, ${ }^{1}$ Rahul Komati, ${ }^{1}$ David A Eichenbaum, ${ }^{2,3}$ Ishani Hariprasad, ${ }^{4}$ \\ Thomas A Ciulla, ${ }^{5}$ Seenu M Hariprasad (D) ${ }^{1}$
}

To cite: Khanna S, Komati R, Eichenbaum DA, et al. Current and upcoming anti-VEGF therapies and dosing strategies for the treatment of neovascular AMD: a comparative review. BMJ Open Ophthalmology 2019;4:e000398. doi:10.1136/ bmjophth-2019-000398

Received 26 September 2019 Revised 5 November 2019 Accepted 21 November 2019

Check for updates

(c) Author(s) (or their employer(s)) 2019. Re-use permitted under CC BY-NC. No commercial re-use. See rights and permissions. Published by BMJ.

${ }^{1}$ Ophthalmology and Visual Sciences, University of Chicago, Chicago, Illinois, USA

${ }^{2}$ Retina Vitreous Associates of Florida, St. Petersburg, Florida, USA

${ }^{3}$ USF Health Morsani College of Medicine, Tampa, Florida, USA

${ }^{4}$ University of Chicago

Laboratory Schools, Chicago,

Illinois, USA

${ }^{5}$ Midwest Eye Institute,

Indianapolis, Indiana, USA

Correspondence to Dr Seenu M Hariprasad; Retina@uchicago.edu

\section{ABSTRACT}

Age-related macular degeneration is the leading cause of vision loss in the developed world, with the expected number of affected elderly individuals reaching 17.8 million. Antivascular endothelial growth factor (anti-VEGF) injection therapy has been instrumental in treating a disease process that was previously thought to be untreatable. Over the past two decades, landmark studies have demonstrated the efficacy of different antiVEGF medications and investigated the optimal dosing regimen and delivery mechanism to increase overall vision and minimise patient burden. In this review, we outline landmark neovascular age-related macular degeneration clinical trials that have demonstrated level 1 evidence for its usage or have contributed to the understanding of how to dose these agents.

\section{INTRODUCTION}

Age-related macular degeneration (AMD) is the leading cause of vision loss in the elderly population in developed countries. ${ }^{12}$ By 2050, the number of individuals affected by AMD is expected to reach 17.8 million, thus demanding the attention of all eye care providers. Neovascular AMD (nAMD) is an advanced stage of AMD characterised by choroidal neovascularisation (CNV) that causes bleeding, fluid accumulation and fibrosis within the macula.

Over the course of the past two decades, there have been revolutionary advances in the treatment of this disease that previously had only limited treatments like destructive thermal laser. ${ }^{3}$ Following Food and Drug Administration (FDA) approval in 1999, verteporfin photodynamic therapy was used to decrease the rate of vision loss, but visual acuity stabilisation or improvement was rare. In the early 2000s, there were major advances in the development of therapies that target vascular endothelial growth factor (VEGF), which plays a key role in both angiogenesis and vascular permeability. ${ }^{4}$ The VEGF family of proteins includes VEGF-A, VEGF-B, VEGF-C, VEGF-D and VEGF-E, and placental growth factor. ${ }^{5}$ The actions of VEGF family members are mediated by the activation of tyrosine-kinase receptors. In 2004, pegaptanib sodium (Macugen; Eyetech Pharmaceuticals, New York, New York), an aptamer designed to target the 165 isoform of VEGF-A, was approved by the FDA, ushering in the new era of anti-VEGF therapy. Registration studies (VISION-1, VEGF Inhibition Study in Ocular Neovascularization-1) showed that subjects receiving intravitreal $0.3 \mathrm{mg}$ pegaptanib every 6 weeks for 1 year experienced approximately half the vision loss as those subjects who received sham. ${ }^{6}$

Pegaptanib has since fallen out of favour after other anti-VEGF agents demonstrated meaningful improvement in vision, including ranibizumab (Lucentis; Genentech, South San Francisco, California), bevacizumab (Avastin; Genentech) and aflibercept (Eylea; Regeneron, Tarrytown, New York). Ranibizumab is a humanised IgG1 antibody fragment against VEGF-A, while bevacizumab is a recombinant humanised monoclonal IgG1 antibody against VEGF-A. Aflibercept is a recombinant protein created by fusing the second immunoglobulin domain of human VEGF receptor 1 with the third domain of human VEGF receptor 2, which in turn is fused to the constant region of human IgG1.

The initial pivotal randomised controlled trials supported monthly dosing for ranibizumab and bimonthly dosing after 3-monthly doses for aflibercept. In order to mitigate the treatment burden of nAMD, attention has been placed on researching the optimal dosing regimen for these medications. AntiVEGF therapy has been administered at regularly spaced fixed intervals in 'continuous' regimens or at varying intervals in 'discontinuous' regimens in an attempt to 
reduce the burden, risks and costs of repeated intravitreal injections. These discontinuous regimens include a "pro re nata' (PRN) approach based on findings of exudation, or a 'treat and extend' (T\&E) approach that gradually increases assessment and treatment intervals after exudation is controlled. However, recent real-world data have shown that patients who receive a low number of annual injections achieve meaningfully worse visual acuity outcomes than those in pivotal trials. ${ }^{7-11}$ In this paper, we will review landmark clinical trials using anti-VEGF injections in the treatment of nAMD. This review will only include studies of currently approved agents, studies of agents with level 1 evidence or studies of agents likely to undergo review by regulatory authorities in the near future. Specifically, this review will evaluate the efficacy of various dosing regimens and examine how they correlate to visual acuity outcomes at 1 year. Efficacy beyond 1 year and safety will not be reviewed, unless these significantly alter conclusions.

\section{Monthly and bimonthly fixed treatment}

Monthly (treatment every 4 weeks) and bimonthly (treatment every 8 weeks after 3 -month loading) fixed regimens were used in the registration trials for ranibizumab and aflibercept, respectively. Consequently, these continuous fixed frequent regimens represent on-label standards of comparison for other discontinuous regimens.

The MARINA (Minimally classic/occult trial of the Anti-VEGF antibody Ranibizumab In the treatment of Neovascular AMD) trial was a 2-year, multicentre, prospective, double-blind trial in which 716 subjects with nAMD with non-classical $\mathrm{CNV}$ were randomised to receive sham injections $(\mathrm{n}=238), 0.3 \mathrm{mg}$ ranibizumab $(\mathrm{n}=238)$ or $0.5 \mathrm{mg}$ ranibizumab $(\mathrm{n}=240)$ injections every 4 weeks for a total of 2 years. ${ }^{12}$ The primary endpoint analysis assessed the superiority of ranibizumab versus sham control at 12 months, with respect to the proportion of subjects losing $<15$ early treatment of diabetic retinopathy (ETDRS) letters of best corrected visual acuity (BCVA). At 12 months, $95 \%$ of the $0.5 \mathrm{mg}$ ranibizumab group (ultimately approved dose) lost $<15$ ETDRS letters, compared with $62 \%$ in the untreated control group. Most importantly, MARINA was one of the two pivotal trials that marked the beginning of vision-improving antiVEGF therapy; at 12 months, the mean BCVA increased 7.2 ETDRS letters from baseline in the $0.5 \mathrm{mg}$ ranibizumab group, whereas the sham injection group lost 10.4 ETDRS letters $(\mathrm{p}<0.0001)$. MARINA demonstrated that monthly $0.5 \mathrm{mg}$ dosing was an effective strategy to improve BCVA in subjects with nAMD with non-classical neovascularisation. In addition, MARINA, conducted in 2003, was the last major anti-VEGF registration trial in nAMD to employ sham control.

ANCHOR (ANti-VEGF Antibody for the Treatment of Predominantly Classic CHORoidal Neovascularization in AMD) was a 2-year, international, multicentre, double-blind study in which 423 subjects with nAMD with predominantly classic $\mathrm{CNV}$ were randomised to receive ranibizumab $0.3 \mathrm{mg}$ plus sham verteporfin therapy, ranibizumab $0.5 \mathrm{mg}$ plus sham verteporfin therapy, or sham injections plus active verteporfin therapy every 4 weeks. ${ }^{13-15}$ Similar to MARINA, the primary endpoint analysis assessed the superiority of ranibizumab versus control at 12 months, with respect to the proportion of subjects losing < 15 ETDRS letters of BCVA; at 12 months, $96 \%$ of the $0.5 \mathrm{mg}$ ranibizumab group lost $<15$ ETDRS letters, compared with $64 \%$ in the verteporfin-treated group. ANCHOR, along with MARINA, shared in the beginning of vision-improving anti-VEGF therapy, as the mean BCVA increased by 11.3 ETDRS letters in the $0.5 \mathrm{mg}$ (ultimately approved dose) ranibizumab group, whereas the verteporfin group decreased by 9.8 ETDRS letters at 12 months $(p<0.001)$. ANCHOR demonstrated that monthly $0.5 \mathrm{mg}$ ranibizumab was an effective, safe and superior treatment to verteporfin in patients with nAMD with classic CNV.

The VEGF Trap Eye: Investigation of Efficacy and Safety in Wet AMD studies (VIEW 1 and VIEW 2) were doubleblind, international, parallel, randomised controlled clinical trials. There were 1217 participants in VIEW 1 and 1240 in VIEW 2. The subjects were randomised to four different regimens: $0.5 \mathrm{mg}$ aflibercept every 4 weeks $(\mathrm{n}=304, \mathrm{n}=311) ; 2 \mathrm{mg}$ aflibercept every 4 weeks $(\mathrm{n}=304$, $\mathrm{n}=313) ; 2 \mathrm{mg}$ every 8 weeks after 3-monthly injections $(\mathrm{n}=303, \mathrm{n}=313)$; or $0.5 \mathrm{mg}$ ranibizumab every 4 weeks $(\mathrm{n}=303, \mathrm{n}=306)$ for the first 52 weeks and then a modified quarterly dosing regimen until 96 weeks. ${ }^{16}$ The primary endpointanalysis assessed non-inferiority (margin of 10\%) of the aflibercept regimens versus monthly ranibizumab in the proportion of subjects losing $<15$ ETDRS letters at month 12. All aflibercept groups were non-inferior to monthly ranibizumab for the primary endpoint (the $2 q 4$, $0.5 q 4$ and 2 q 8 regimens were $95.1 \%, 95.9 \%$ and $95.1 \%$, respectively, for VIEW 1 , and $95.6 \%, 96.3 \%$ and $95.6 \%$, respectively, for VIEW 2, whereas monthly ranibizumab was $94.4 \%$ in both studies). In the integrated analysis of the two trials, there was no statistically significant change in the improvement of ETDRS letters at 1 year in each of the four groups. The VIEW trials demonstrated that $2 \mathrm{mg}$ aflibercept dosed every 8 weeks (after 3-monthly loading injections) was non-inferior to $0.5 \mathrm{mg}$ ranibizumab dosed every 4 weeks.

\section{PRN treatment}

The PrONTO (Prospective Optical Coherence Tomography (OCT) Imaging of Patients with Neovascular AMD Treated with intra-Ocular Ranibizumab) study was a 2-year, prospective, single-centre study in which 40 subjects were treated with monthly $0.5 \mathrm{mg}$ injections of ranibizumab for three consecutive months and re-evaluated for subsequent injections based on five different criteria on time-domain OCT. The criteria include BCVA loss of a minimum of five ETDRS letters with OCT evidence of fluid in the macula, an increase in OCT central retinal thickness (CRT) of $100 \mu \mathrm{m}$, macular haemorrhage, new area of CNV and evidence of persistent fluid on OCT 
Table 1 Study, dosing regimen, number of injections and change in ETDRS letters

\begin{tabular}{|c|c|c|c|c|c|}
\hline Study & Regimen & Drug & $\begin{array}{l}\text { Baseline BCVA } \\
\text { (ETDRS Letter) }\end{array}$ & $\begin{array}{l}\text { \# Injections } \\
\text { (1 year) }\end{array}$ & $\begin{array}{l}\text { Change in ETDRS } \\
\text { Letter (1 year) }\end{array}$ \\
\hline ANCHOR & q4 & Ranibizumab & 53.7 & 11.2 & 11.3 \\
\hline MARINA & q4 & Ranibizumab & 47.1 & 12.3 & 7.2 \\
\hline VIEW 1 & q4 & Aflibercept & 55.2 & 12.3 & 10.9 \\
\hline VIEW 1 & q8 & Aflibercept & 55.7 & 7.5 & 7.9 \\
\hline VIEW 1 & $q 4$ & Ranibizumab & 54 & 12.3 & 8.1 \\
\hline VIEW 2 & $q 4$ & Aflibercept & 52.8 & 12.3 & 7.6 \\
\hline VIEW 2 & q8 & Aflibercept & 51.6 & 7.5 & 8.9 \\
\hline VIEW 2 & q4 & Ranibizumab & 53.8 & 12.3 & 9.4 \\
\hline PRONTO & PRN & Ranibizumab & 56.2 & 5.3 & 9.6 \\
\hline SUSTAIN & PRN & Ranibizumab & 56.1 & 5.6 & 3.6 \\
\hline HARBOR & $\mathrm{q} 4$ & Ranibizumab & 54.2 & 11.3 & 10.1 \\
\hline HARBOR & PRN & Ranibizumab & 54.5 & 7.7 & 8.2 \\
\hline CATT & q4 & Bevacizumab & 60.2 & 11.9 & 8 \\
\hline CATT & PRN & Bevacizumab & 60.4 & 7.7 & 5.9 \\
\hline CATT & PRN & Ranibizumab & 61.5 & 6.9 & 6.8 \\
\hline CATT & q4 & Ranibizumab & 60.1 & 11.7 & 8.5 \\
\hline IVAN & q4 & Bevacizumab & 60 & 12 & 6.8 \\
\hline IVAN & PRN & Mixed & 62.9 & 7 & 5.5 \\
\hline PIER & $\mathrm{q} 12$ & Ranibizumab & 53.7 & 6 & -0.2 \\
\hline EXCITE & $\mathrm{q} 12$ & Ranibizumab & 57.5 & 5.5 & 3.8 \\
\hline HAWK & $q 12 / q 8$ & Brolucizumab (3 mg) $\dagger$ & 60.6 & $6.7^{*}$ & 6.1 \\
\hline HAWK & $q 12 / q 8$ & Brolucizumab (6 mg) & 60.6 & $6.6^{*}$ & 6.6 \\
\hline HAWK & q8 & Aflibercept & 60.6 & 8 & 6.8 \\
\hline HARRIER & $q 12 / q 8$ & Brolucizumab (6 mg) & 61.2 & $6.7^{*}$ & 6.9 \\
\hline HARRIER & q8 & Aflibercept & 61.2 & 8 & 7.6 \\
\hline LUCAS & T\&E & Ranibizumab & 61.6 & 8 & 8.2 \\
\hline LUCAS & T\&E & Bevacizumab & 59.6 & 8.9 & 7.9 \\
\hline TREX-AMD & q4 & Ranibizumab & 60.3 & 13 & 9.2 \\
\hline TREX-AMD & T\&E & Ranibizumab & 59.9 & 10.1 & 10.5 \\
\hline TREND & T\&E & Ranibizumab & 59.5 & 8.7 & 6.2 \\
\hline TREND & $q 4$ & Ranibizumab & 60.6 & 11.1 & 8.1 \\
\hline CEDAR & q8 & Abicipar & 56 & 8 & 6.7 \\
\hline CEDAR & $q 12$ & Abicipar & 56 & 6 & 5.6 \\
\hline CEDAR & $\mathrm{q} 4$ & Ranibizumab & 56 & 13 & 8.5 \\
\hline SEQUOIA & q8 & Abicipar & 57.2 & 8 & 8.3 \\
\hline SEQUOIA & q12 & Abicipar & 56.4 & 6 & 7.3 \\
\hline SEQUOIA & $q 4$ & Ranibizumab & 57.1 & 13 & 8.3 \\
\hline
\end{tabular}

*Extrapolated based on the study design given variable dosing in single study arm with weighted average based on minimum number of injections received (6) and maximum average injections received (7.5). Bolded studies are level 1 FDA registration and National Eye Institute trials which are included in the analysis.

†Brolucizumab $3 \mathrm{mg}$ dose is the triangle data point in figure 1.

ANCHOR, Anti-VEGF Antibody for the Treatment of Predominantly Classic CHORoidal Neovascularization in AMD; BCVA, best corrected visual acuity; CATT, Comparison of Age-Related Macular Degeneration Treatments Trials; ETDRS, early treatment of diabetic retinopathy; EXCITE,

Efficacy and Safety of Ranibizumab in subjects with Subfoveal and CNV secondary to AMD; FDA, Food and Drug Administration; LUCAS, Lucentis Compared to Avastin Study; MARINA, Minimally classic/occult trial of the Anti-VEGF antibody Ranibizumab In the treatment of Neovascular AMD; PRN, pro re nata; PrONTO, Prospective Optical Coherence Tomography (OCT) Imaging of Patients with Neovascular AMD Treated with intra-Ocular Ranibizumab; q4, every 4 weeks; q8, every 8 weeks; q12, every 12 weeks; SUSTAIN, Study of Ranibizumab in Patients With Subfoveal Choroidal Neovascularization Secondary to Age-Related Macular Degeneration; T\&E, treat and extend; TREX-AMD, Treat and Extend Protocol in Patients with Wet Age-Related Macular Degeneration; VIEW, The VEGF Trap Eye: Investigation of Efficacy and Safety in Wet AMD study. 
1 month after prior injection. The criteria were changed in the second year to include any qualitative increase in fluid on OCT. At 12 months, the mean number of injections received was 5.6 with a gain of 9.3 ETDRS letters $(\mathrm{p}<0.001) .{ }^{17} 18$ These BCVA results compare favourably with ANCHOR and MARINA, in which many more injections were administered, but PrONTO was a small, single-centre, uncontrolled, open-label study, and consequently the efficacy results must be interpreted in this context. Nevertheless, PrONTO is important because it supported a role for OCT-guided anti-VEGF treatment in nAMD.

The SUSTAIN (Study of Ranibizumab in Patients with Subfoveal Choroidal Neovascularization Secondary to Age-Related Macular Degeneration) trial was a prospective, ex-US multicentre, year-long study evaluating the $0.3 \mathrm{mg}$ ranibizumab regimen in both classic and nonclassic nAMD. ${ }^{19}$ Of the participants, $12 \%$ received $0.5 \mathrm{mg}$ dose after approval by the European Medicines Agency. Five hundred and thirty-one subjects received 3-monthly injections of $0.3 \mathrm{mg}$ or $0.5 \mathrm{mg}$ ranibizumab and received the injection only if one of the following criteria was met: more than five-letter loss in the BCVA from the previous highest BCVA in the first 3 months, or $100 \mu \mathrm{m}$ increase in CRT from the previous lowest measurement in the first 3 months. In the study, the mean BCVA initially improved by 5.8 ETDRS letters after the three loading doses but decreased to 3.6 ETDRS letters at month 12 with a mean of 5.6 injections.

HARBOR (pHase III, double-masked, multicenter, randomized, Active treatment-controlled study of the efficacy and safety of $0.5 \mathrm{mg}$ and $2.0 \mathrm{mg}$ Ranibizumab administered monthly or on an as-needed Basis (PRN) in patients with subfoveal neOvasculaR age-related macular degeneration) was a multicentre, double-masked, doseresponse, active-controlled study in which subjects with subfoveal nAMD $(\mathrm{n}=1098)$ were randomised to receive (1) ranibizumab $0.5 \mathrm{mg}$ every 4 weeks $(\mathrm{n}=276)$ or PRN following three initial monthly injections $(n=275)$; or (2) ranibizumab $2 \mathrm{mg}$ every 4 weeks $(\mathrm{n}=274)$ or $\mathrm{PRN}$ following three initial monthly injections $(\mathrm{n}=273)$. An injection was administered if there was greater than five-letter decrease in BCVA from previous visit or any evidence of disease on OCT. ${ }^{20}{ }^{21}$ The mean improvements in BCVA (ETDRS letters) at month 12 were 10.1 (0.5 mg monthly, 11.3 injections), 8.2 (0.5 mg PRN, 7.7 injections), 9.2 (2.0 mg monthly, 11.2 injections) and 8.6 (2.0 mg PRN, 6.9 injections). There was no evidence that the $2.0 \mathrm{mg}$ monthly dosing regimen was superior to the $0.5 \mathrm{mg}$ monthly regimen, while the $0.5 \mathrm{mg}$ and $2.0 \mathrm{mg}$ PRN regimens did not meet the four-letter non-inferiority margin compared with the $0.5 \mathrm{mg}$ monthly regimen. Overall, the monthly regimens trended towards numerically better visual outcomes.

CATT (Comparison of Age-Related Macular Degeneration Treatments Trials) was a 2-year multicentre trial that randomised 1208 subjects with subfoveal CNV secondary to AMD to one of four groups: (1) ranibizumab on a fixed schedule of every 4 weeks for 1 year and rerandomisation to every 4 weeks or variable dosing; (2) bevacizumab on fixed schedule of every 4 weeks for a year then randomised to bevacizumab to every 4 weeks or variable dosing; (3) ranibizumab on variable dosing for 2 years; and (4) bevacizumab on variable dosing for 2 years. ${ }^{22} 23$ The primary outcome was BCVA at 1 year with a non-inferiority limit of 5 ETDRS letters. The monthly regimens trended towards better visual outcomes. Ranibizumab and bevacizumab administered monthly led to 8.5 and 8.0 ETDRS letters gained, respectively. Ranibizumab and bevacizumab administered on a PRN basis led to a gain of 6.8 and 5.9 ETDRS letters, respectively. Among subjects following the same regimen for 2 years, the mean gain in BCVA was similar for both drugs but greater for monthly than for PRN treatment (difference, 2.4 ETDRS letters; $\mathrm{p}=0.046$ ).

IVAN (Inhibition of VEGF in Age-related choroidal Neovascularisation) was a multicentre, double-blinded, randomised UK study in which 610 subjects with nAMD were randomised to one of four groups. ${ }^{24}$ The four groups included ranibizumab $0.5 \mathrm{mg}$ on a fixed monthly schedule $(\mathrm{n}=157)$; ranibizumab $0.5 \mathrm{mg}$ three initial monthly injections then PRN $(\mathrm{n}=155)$; bevacizumab $1.25 \mathrm{mg}$ on a fixed monthly schedule $(\mathrm{n}=148)$; or bevacizumab $1.25 \mathrm{mg}$ three initial monthly injections then PRN $(n=145)$. If retreatment was needed, then monthly injections over 3 months were mandated. After 2 years, bevacizumab was neither non-inferior nor inferior to ranibizumab (-1.37 ETDRS letters), and discontinuous treatment was neither non-inferior nor inferior to continuous treatment (-1.63 ETDRS letters).

\section{Quarterly treatment}

PIER (Phase IIIb, multicenter, randomized, doublemasked, sham Injection-controlled study of the Efficacy and safety of Ranibizumab) was a 2-year, multicentre, randomised, double-masked, sham-controlled study in which subjects with subfoveal CNV secondary to AMD $(\mathrm{n}=184)$ were randomised to receive ranibizumab $0.3 \mathrm{mg}$ $(\mathrm{n}=60)$, ranibizumab $0.5 \mathrm{mg}(\mathrm{n}=61)$ or sham injections $(n=63)$ every 3 months following three initial monthly injections. ${ }^{25}$ At 12 months, BCVA decreased in all groups an average of 16.3, 1.6 and 0.2 ETDRS letters for the sham, $0.3 \mathrm{mg}$ and $0.5 \mathrm{mg}$ groups, respectively $(\mathrm{p}<0.0001$, treatment vs sham). Clearly, like the trials before it, there was a benefit from the monthly ranibizumab dosing.

EXCITE (Efficacy and Safety of Ranibizumab in subjects with Subfoveal and CNV secondary to AMD) was an ex-US multicentre, randomised, double-masked, activecontrolled study in which subjects with subfoveal CNV secondary to AMD ( $\mathrm{n}=353$ ) were randomised to three different groups. ${ }^{26}$ The groups included ranibizumab $0.3 \mathrm{mg}$ every 3 months following three initial monthly injections $(\mathrm{n}=120)$; ranibizumab $0.5 \mathrm{mg}$ every 3 months following three initial monthly injections $(n=118)$; or ranibizumab $0.3 \mathrm{mg}$ every 4 weeks $(\mathrm{n}=115)$. This trial corroborated the superiority of monthly dosing. In the intent-to-treat population using last observation carried 
forward, monthly dosing resulted in a visual gain of 8.0 ETDRS letters compared with 4.0 and 2.8 ETDRS letters for the $0.3 \mathrm{mg}$ and $0.5 \mathrm{mg}$ quarterly injection groups, respectively.

\section{Upcoming treatments using quarterly dosing}

More recently, brolucizumab (Novartis, Basel, Switzerland) has been FDA-approved in October 2019 after an expedited review of phase III data. Brolucizumab (Novartis) is a $26 \mathrm{kDa}$, humanised single-chain antibody fragment that, like existing anti-VEGF therapy, targets VEGF-A. The HAWK (Efficacy and Safety of RTH258 versus Aflibercept Study 1)and HARRIER (Efficacy and Safety of RTH258 versus Aflibercept Study 2) studies are 96-week, double-blind, multicentre, phase III clinical trials investigating brolucizumab $6 \mathrm{mg}$ (HAWK and HARRIER) and brolucizumab $3 \mathrm{mg}$ (HAWK) versus aflibercept $2 \mathrm{mg}$ in 1817 subjects with untreated nAMD ${ }^{27}{ }^{28}$ In these studies, there was a 3-month loading phase, followed by dosing every 12 weeks for the brolucizumab groups, with an option to decrease to 8-week dosing based on evidence of disease activity; aflibercept was dosed bimonthly per its label. At 2 years, brolucizumab demonstrated non-inferiority in BCVA compared with aflibercept with a similar safety profile. There was an improvement of 6.6 and 6.1 ETDRS letters with brolucizumab $6 \mathrm{mg}$ and $3 \mathrm{mg}$, respectively, vs 6.8 ETDRS letters with aflibercept in HAWK $(\mathrm{p}<0.001)$ and 6.9 ETDRS letters with brolucizumab $6 \mathrm{mg}$ vs 7.6 ETDRS letters with aflibercept in HARRIER $(p<0.001)$. More than half of brolucizumab $6 \mathrm{mg}$ eyes were maintained on dosing every 12 weeks through 48 weeks. ${ }^{29}$ Based on these two trials, brolucizumab may have similar efficacy compared with aflibercept while providing some relief in injection treatment frequency.

Abicipar pegol (Allergan, Dublin, Ireland) is a DARPinbased (designed ankyrin repeat protein) anti-VEGF drug being evaluated for the treatment of nAMD. DARPin therapeutics are a class of small proteins derived from natural ankyrin repeat proteins, which bind to a single target. The CEDAR (Safety and Efficacy of Abicipar Pegol in Patients with Neovascular AMD NCT02462928) and SEQUOIA (Safety and Efficacy of Abicipar Pegol in Patients with Neovascular AMD NCT02462486) trials are 96-week, phase III, multicentre, randomised control trials comparing three different groups $(n=817, n=831)$ : abicipar pegol $2 \mathrm{mg}$ monthly for the first 3 months, followed by injections every 8 weeks $(n=265, n=267)$; abicipar pegol $2 \mathrm{mg}$ on day 1 , week 4 and week 12 , followed by injections every 12 weeks $(n=262, n=265)$; and ranibizumab $0.5 \mathrm{mg}$ every 4 weeks $(\mathrm{n}=290, \mathrm{n}=299) .{ }^{30}$ The primary endpoint at 1 year was non-inferiority to ranibizumab, which was met. The mean change in BCVA for the three groups was 6.7, 5.6 and 8.5 in CEDAR, respectively, while the mean change in BCVA was 8.3, 7.3 and 8.3 in SEQUOIA, respectively. Most notable was the difference in the safety profile of the comparable agents showing a $15 \%$ intraocular inflammatory event rate in the abicipar groups compared with $<1 \%$ for the ranibizumab arm in both trials. Recently, the MAPLE (Evaluating Abicipar for Safety and Treatment Effect in Patients with Neovascular AMD) trial, a 28-week, open-label trial which enrolled 123 subjects who received 3-monthly $2 \mathrm{mg}$ abicipar injections followed by $2 \mathrm{mg}$ injections every 8 weeks for a total of five injections through 28 weeks, revealed a lower incidence of intraocular inflammation (8.9\%) after changes to the manufacturing process. In MAPLE the incidence of severe inflammation was $1.6 \%$, with one reported case of iritis and one reported case of uveitis. ${ }^{31}$

\section{Treat and extend}

The LUCAS (Lucentis Compared to Avastin Study) protocol was a Norwegian, multicentre, randomised, noninferiority study in which subjects with nAMD $(n=371)$ were randomised to receive either ranibizumab $0.5 \mathrm{mg}$ $(\mathrm{n}=187)$ or bevacizumab $1.25 \mathrm{mg}(\mathrm{n}=184)$ on a monthly basis until inactive disease was established. At that point, the treatment interval was extended by 2 weeks at a time. The increase in BCVA was 8.2 ETDRS letters with 8.0 injections in the ranibizumab group and 7.9 ETDRS letters with 8.9 injections in the bevacizumab group ( $\mathrm{p}=0.845$ for ETDRS letter, $\mathrm{p}=0.020$ for injections). ${ }^{32} 33$ At 2 years, although bevacizumab and ranibizumab had an equivalent effect on BCVA administered according to a T\&E regimen, there was a statistically significant difference between the drugs regarding the number of treatments administered (18 vs 16 , respectively).

TREX-AMD (Treat and Extend Protocol in Patients with Wet Age-Related Macular Degeneration) was a twocentre, randomised controlled study in which subjects with nAMD $(\mathrm{n}=60)$ were randomised to receive ranibizumab $0.5 \mathrm{mg}$ every 4 weeks $(\mathrm{n}=20)$ or ranibizumab $0.5 \mathrm{mg}$ using a T\&E protocol following three initial monthly injections $(n=40) .{ }^{34}$ Subjects in the TREX (Treat and Extend) cohort were treated based on exudative disease activity; however, they were not treated less frequently than every 12 weeks. The intervals between each study visit were lengthened or reduced by 2 weeks based on whether OCT revealed exudative activity. At 12 months, the monthly group noted an improvement of 9.2 ETDRS letters, while the T\&E group demonstrated a mean improvement of 10.5 ETDRS letters $(p=0.60)$. The mean number of injections was 13 and 10.1 for the groups, respectively $(\mathrm{p}<0.0001) .^{35}$ This study suggests that similar BCVA can be maintained with slightly fewer injections using the T\&E protocol.

The TREND (TReat and extEND) study was an ex-US multicentre, randomised controlled study in which subjects with nAMD $(\mathrm{n}=650)$ were randomised to receive $0.5 \mathrm{mg}$ ranibizumab monthly $(\mathrm{n}=327)$ or $0.5 \mathrm{mg}$ ranibizumab using a T\&E protocol $(n=323)$ and is one of the largest studies that compare the T\&E regimen with monthly dosing. ${ }^{36}$ The subjects in the T\&E group initially were given 2-monthly injections and subsequently received injections based on disease activity by BCVA and OCT criteria. If no disease activity was present, the 
interval between treatments was extended by 2 weeks, with a minimum of 4 weeks between treatments and a maximum of 12 weeks. The BCVA change was 6.2 ETDRS letters in the T\&E group (8.7 injections) and 8.1 ETDRS letters in the monthly group (11.1 injections) $(\mathrm{p}<0.001$ for non-inferiority), demonstrating a clinically comparable outcome for the T\&E regimen.

\section{DISCUSSION}

Registration studies such as ANCHOR/MARINA and VIEW 1/2 demonstrated the effectiveness of ranibizumab and aflibercept, respectively, when administered continuously at fixed frequent intervals. However, given the treatment burden with monthly or bimonthly fixedinterval treatment, there has been great interest in alternative dosing regimens. PIER and EXCITE assessed quarterly dosing after 3-monthly doses of ranibizumab and validated better visual gains for patients receiving monthly injections. There were two interesting observations from the quarterly dosing patients: (1) in EXCITE, patients trended towards mean deterioration in BCVA and macular thickness change between quarterly treatments, with 'sawtooth' patterns observed when these parameters were graphed over time, and (2) about half to two-thirds of patients who received quarterly dosing maintained or gained vision. ${ }^{37}$ It is likely that the mean vision in the quarterly treatment groups in both of these studies was reduced by a proportion of patients doing poorly with quarterly dosing. Subjects in PIER were both monitored and treated quarterly. Subjects in EXCITE were monitored monthly but treated quarterly, which likely led to the relatively better mean visual performance versus PIER, since patients with disease activity detected with more frequent observation exited the study, likely at investigator discretion. ${ }^{37}$

Given these results with quarterly dosing, interest has shifted to variable frequency anti-VEGF treatment regimens, which has now seen widespread adoption. Specifically, the 2019 American Society of Retina Specialists Preferences and Trends Survey of more than 1000 responding retina specialists in 60 countries revealed that more than $98 \%$ of retina specialists, both in the USA and internationally, use some form of OCT-guided variable frequency anti-VEGF treatment protocols for nAMD. ${ }^{38}$ However, attempts to limit treatment burden through the adoption of variable frequency anti-VEGF treatment regimens must be approached cautiously. For example, SUSTAIN did not corroborate the impressive visual gain observed in PrONTO using an OCT-guided PRN dosing regimen, while HARBOR and CATT demonstrated that a PRN dosing regimen results in a less favourable visual outcome compared with fixed, frequent anti-VEGF suppression.

'Treat-and-extend', a commonly used approach, ${ }^{37}$ seemed to perform relatively well in LUCAS, TREX and TREND. Although these studies suggest that similar BCVA can be maintained with slightly fewer injections, the number of treatments exceeded eight in the first year.
It seems apparent that, regardless of regimen, relatively frequent treatment of eyes with nAMD is required for good vision with current commercially available agents. This supposition is supported by an analysis of the relationship between the number of anti-VEGF injections and letters gained (table 1, figure 1). This analysis substantiates the doctrine of 'more injections yields better vision', at least with regard to current commercially available agents, and is consistent with a previous report. ${ }^{39}$

However, real-world studies suggest that patients are undertreated. $^{7-11}$ In one recent study of 49485 eyes, patients with nAMD received a mean of 7.3 anti-VEGF injections, resulting in a mean gain of only one letter $(\mathrm{p}<0.001)$. Long-term visual outcomes are even worse. ${ }^{8}$ The SEVEN-UP (Seven Year Observational Update of Macular Degeneration Patients Post-MARINA/ANCHOR and HORIZON Trials) study (2013) examined long-term data from ANCHOR and MARINA trial patients, who were managed per the discretion of their primary ophthalmologist. ${ }^{40}$ Data from 65 patients were collected at 7.3 years after initial enrolment in the ANCHOR/MARINA trials and 3.4 years after completion of the HORIZON (An Open-Label Extension Trial of Ranibizumab for Choroidal Neovasculariation Secondary to Age-Related macular degeneration) extension study. There was an average loss of 8.6 ETDRS letters $(p<0.005)$ from baseline at entry of ANCHOR/MARINA trials and loss of 6.9 ETDRS letters from HORIZON month $24(\mathrm{p}<0.001)$. The mean number of injections was 6.8 in the 3.4 years after completing HORIZON.

In using our commercially available agents, there seems to be trouble generating a 'one size fits all' approach to nAMD therapy. This is most likely due to patient factors over therapeutic factors, since our existing commercially available therapeutics are similar. We know from registration data, extension data and PRN data that a relatively frequent treatment plan is appropriate for the average patient or average patient population. On a discrete patient-to-patient level in the real world, frequent anatomical assessment with OCT with some individualisation of dosing is an appropriate plan to mitigate treatment burden as much as possible.

Nevertheless, the future for nAMD treatment is promising. New longer acting agents such as brolucizumab and abicipar have shown efficacy at 12-week dosing intervals through HAWK/HARRIER and CEDAR/ SEQUOIA trials, respectively. Sustained-release antiVEGF treatments, to address treatment burden, include the ranibizumab port delivery system, KSI-301 and GB-102. ${ }^{41-43}$ Other unique anti-VEGF agents in current trials include conbercept, a fusion protein of the VEGF receptor domains, and OPT-302, an inhibitor of VEGF-C/D. ${ }^{44}$ Strategies to activate the Tie-2 receptor are of interest, with recent trials of faricimab, a bispecific antibody targeting both VEGF-A and angiopoietin-2. ${ }^{45}$ While most topical anti-VEGF therapy so far has failed to reduce the need for rescue intravitreal anti-VEGF injections in clinical trials, PAN-90806 is currently being 


\section{Change in BCVA vs number of injections}

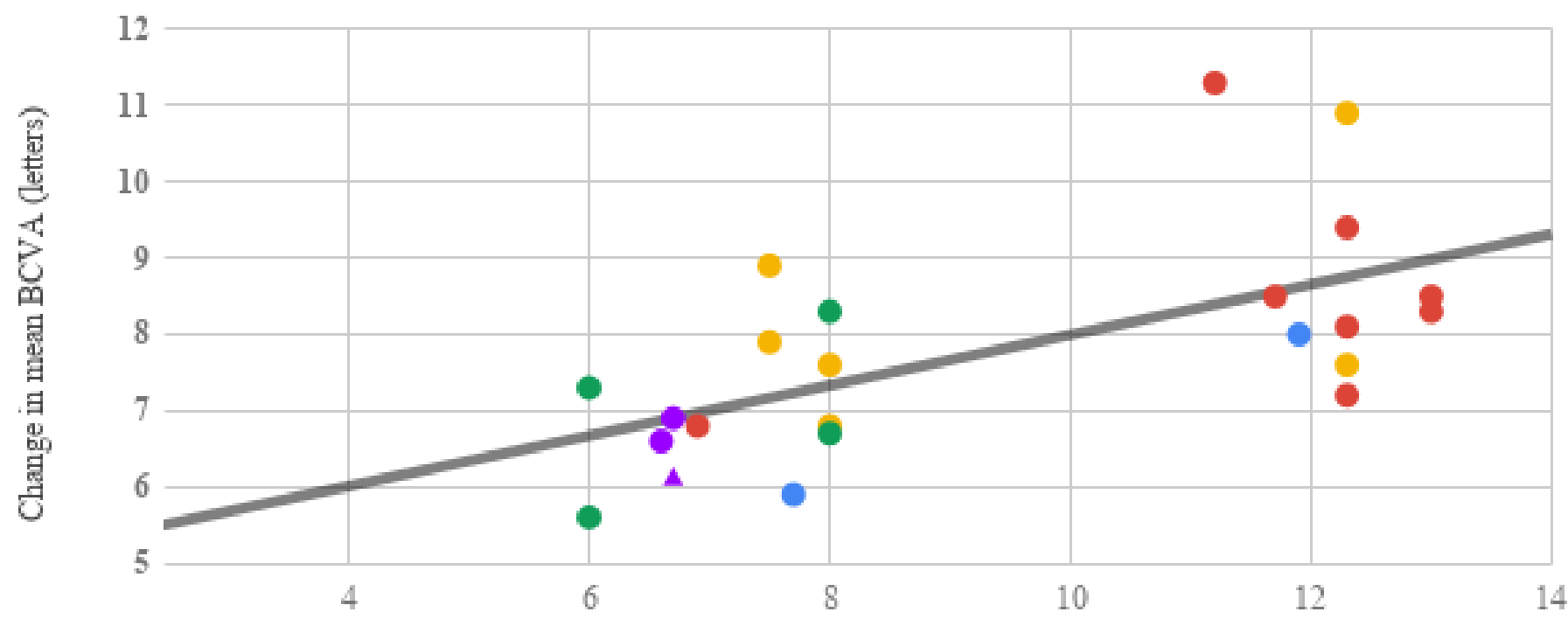

Number of injections in 1 year

\section{Bevacizumab Ranibizumab Aflibercept Abicipar Brolucizumab \\ Change in mean BCVA (1 year) - Trendline for Letters Gained (1 year) $\mathrm{R}^{2}=0.373$}

Figure 1 The figure explores the relationship between the number of injections and the number of ETDRS letters gained. Even though the agents have different durability, there is a positive and clinically meaningful correlation. Only FDA registration and National Eye Institute trials were included in this analysis (bolded in table 1). Brolucizumab $3 \mathrm{mg}$ dose is the triangle data point in the figure. BCVA, best corrected visual acuity; ETDRS, early treatment of diabetic retinopathy; FDA, Food and Drug Administration.

studied in phase I/II trials, with promising initial results. ${ }^{46}$ In theory, gene therapy with viral vectors could represent the ultimate form of sustained anti-VEGF-A treatment, as intraocular tissue could be modified to develop its own anti-VEGF-A secretory mechanism that would diminish the need for intravitreal injections. These gene therapies include RGX-314 and ADVM-022, which are currently in early-phase clinical trials. ${ }^{47}$ In the future, the retina community will have an exciting repertoire of new therapies for nAMD, which will greatly improve the quality of life for countless patients.

Contributors SMH, TAC, DAE conceived and designed the project. SK, RK, SMH were involved in data collection and analysis. SK drafted and revised the manuscript. RK, IH, SMH, TAC, DAE contributed to writing and critical revision of the manuscript. All authors reviewed and edited the final manuscript.

Funding The authors have not declared a specific grant for this research from any funding agency in the public, commercial or not-for-profit sectors.

Competing interests SMH is a consultant or on speaker's bureau for the following companies: Novartis/Alcon, Spark, Regeneron, Alimera Sciences, Clearside Biomedical, EyePoint Pharmaceuticals, OD-OS and Allergan. DAE is a speaker (S), consultant (C), investigator (I) or equity/stockholder (E) in the following companies: Genentech (C, I, S), Regeneron (C), Allergan (C, I, S), Clearside (C, I, E), Novartis (C, I, S), Alimera (C, I), Ophthotech (I), Opthea (I), US Retina (E), Hemera Biopharmaceuticals $(E)$, Boston Image Reading Center $(E)$, Notal Vision $(C, S)$, Allegro (C), EyePoint (C), Mylan (I), Chengdu (I), Orbit Biomedical (C), Adverum (C) and Kodiak (C, I). TAC has an employment relationship with, and equity ownership in, Clearside Biomedical. This work was undertaken in his role as Volunteer Clinical Professor at the Indiana University School of Medicine.
Patient consent for publication Not required.

Provenance and peer review Not commissioned; externally peer reviewed.

Open access This is an open access article distributed in accordance with the Creative Commons Attribution Non Commercial (CC BY-NC 4.0) license, which permits others to distribute, remix, adapt, build upon this work non-commercially, and license their derivative works on different terms, provided the original work is properly cited, appropriate credit is given, any changes made indicated, and the use is non-commercial. See: http://creativecommons.org/licenses/by-nc/4.0/.

ORCID iD

Seenu M Hariprasad http://orcid.org/0000-0003-0985-9551

\section{REFERENCES}

1 Klein R. Prevalence of age-related macular degeneration in the US population. Arch Ophthal 2011;129:75.

2 Klein BEK. Forecasting age-related macular degeneration through 2050. JAMA 2009;301.

3 Fine SL. Macular photocoagulation study. Arch Ophthalmol 1980;98:832.

4 Miller JW, Le Couter J, Strauss EC, et al. Vascular endothelial growth factor A in intraocular vascular disease. Ophthalmology 2013:120:106-14.

5 Holmes DIR, Zachary I. The vascular endothelial growth factor (VEGF) family: angiogenic factors in health and disease. Genome Biol 2005;6:209.

6 Gragoudas ES, Adamis AP, Cunningham ET, et al. Pegaptanib for neovascular age-related macular degeneration. N Engl J Med 2004;351:2805-16.

7 Holz FG, Tadayoni R, Beatty S, et al. Multi-country real-life experience of anti-vascular endothelial growth factor therapy for wet age-related macular degeneration. Br J Ophthalmol 2015;99:220-6.

8 Ciulla TA, Hussain RM, Pollack JS, et al. Visual Acuity Outcomes and Anti-Vascular Endothelial Growth Factor Therapy Intensity in 
Neovascular AMD Patients: A "Real World" Analysis in 49,485 Eyes. Ophthalmology Retina. [Epub ahead of print: May 2019].

9 Ciulla TA, Huang F, Westby K, et al. Real-World outcomes of AntiVascular endothelial growth factor therapy in neovascular agerelated macular degeneration in the United States. Ophthalmology Retina 2018;2:645-53.

10 Rao P, Lum F, Wood K, et al. Real-World vision in age-related macular degeneration patients treated with single Anti-VEGF drug type for 1 year in the iris registry. Ophthalmology 2018;125:522-8.

11 Holekamp NM, Liu Y, Yeh W-S, et al. Clinical utilization of anti-VEGF agents and disease monitoring in neovascular age-related macular degeneration. Am J Ophthalmol 2014;157:825-33.

12 Kaiser PK, Blodi BA, Shapiro H, et al. Angiographic and optical coherence tomographic results of the marina study of ranibizumab in neovascular age-related macular degeneration. Ophthalmology 2007;114:1868-75.

13 Brown DM, Kaiser PK, Michels M, et al. Ranibizumab versus verteporfin for neovascular age-related macular degeneration. $N$ Engl J Med 2006;355:1432-44.

14 Kaiser PK, Brown DM, Zhang K, et al. Ranibizumab for predominantly classic neovascular age-related macular degeneration: subgroup analysis of first-year anchor results. Am J Ophthalmol 2007;144:850-7.

15 Brown DM, Michels M, Kaiser PK, et al. Ranibizumab versus verteporfin photodynamic therapy for neovascular age-related macular degeneration: two-year results of the anchor study. Ophthalmology 2009;116:57-65.

16 Heier JS, Brown DM, Chong V, et al. Intravitreal aflibercept (VEGF Trap-Eye) in wet age-related macular degeneration. Ophthalmology 2012;119:2537-48.

17 Fung AE, Lalwani GA, Rosenfeld PJ, et al. An optical coherence Tomography-Guided, variable dosing regimen with intravitreal ranibizumab (Lucentis) for neovascular age-related macular degeneration. Am J Ophthalmol 2007;143:566-83.

18 Lalwani GA, Rosenfeld PJ, Fung AE, et al. A variable-dosing regimen with intravitreal ranibizumab for neovascular age-related macular degeneration: year 2 of the PrONTO study. Am J Ophthalmol 2009;148:43-58

19 Holz FG, Amoaku W, Donate J, et al. Safety and efficacy of a flexible dosing regimen of ranibizumab in neovascular agerelated macular degeneration: the sustain study. Ophthalmology 2011;118:663-71.

20 Busbee BG, Ho AC, Brown DM, et al. Twelve-Month efficacy and safety of $0.5 \mathrm{Mg}$ or $2.0 \mathrm{Mg}$ ranibizumab in patients with subfoveal neovascular age-related macular degeneration. Ophthalmology 2013;120:1046-56.

21 AC H, Busbee BG, Regillo CD, et al. Twenty-four-Month efficacy and safety of $0.5 \mathrm{Mg}$ or $2.0 \mathrm{Mg}$ ranibizumab in patients with subfoveal neovascular age-related macular degeneration. Ophthalmology 2014;121:2181-92.

22 Martin DF, Maguire MG, CATT Research Group,. et al. Ranibizumab and bevacizumab for neovascular age-related macular degeneration. N Engl J Med 2011;364:1897-908.

23 Martin DF, Maguire MG, Fine SL, et al. Ranibizumab and bevacizumab for treatment of neovascular age-related macular degeneration. Ophthalmology 2012;119:1388-98.

24 Chakravarthy U, Harding SP, Rogers CA, et al. A randomised controlled trial to assess the clinical effectiveness and costeffectiveness of alternative treatments to inhibit VEGF in agerelated choroidal neovascularisation (IVAN). Health Technol Assess 2015;19:1-298.

25 Regillo CD, Brown DM, Abraham P, et al. Randomized, doublemasked, sham-controlled trial of ranibizumab for neovascular agerelated macular degeneration: PIER study year 1. Am J Ophthalmol 2008;145:239-48.

26 Schmidt-Erfurth U, Eldem B, Guymer R, et al. Efficacy and safety of monthly versus Quarterly ranibizumab treatment in neovascular age-related macular degeneration: the excite study. Ophthalmology 2011;118:831-9.
27 Efficacy and safety of RTH258 versus aflibercept. Available: https:// clinicaltrials.gov/ct2/show/NCT02307682 [Accessed 14 Apr 2019].

28 Efficacy and Safety of RTH258 Versus Aflibercept - Study 2. Available: https://clinicaltrials.gov/ct2/show/NCT02434328

29 Dugel PU, Koh A, Ogura Y, et al. Hawk and harrier: phase 3 , multicenter, randomized, double-masked trials of Brolucizumab for neovascular age-related macular degeneration. Ophthalmology 2019. doi:10.1016/j.ophtha.2019.04.017. [Epub ahead of print: Apr 2019].

30 Khurana R. Safety and efficacy of abicipar in patients with neovascular age-related macular degeneration 2018.

31 Molecular Partners. Allergan and molecular partners announce Topline safety results from maple study of Abicipar pegol 2019;2019.

32 Berg K, Pedersen TR, Sandvik L, et al. Comparison of ranibizumab and bevacizumab for neovascular age-related macular degeneration according to LUCAS Treat-and-Extend protocol. Ophthalmology 2015;122:146-52.

33 Berg K, Hadzalic E, Gjertsen I, et al. Ranibizumab or bevacizumab for neovascular age-related macular degeneration according to the Lucentis compared to Avastin study Treat-and-Extend protocol. Ophthalmology 2016;123:51-9.

34 Wykoff CC, Croft DE, Brown DM, et al. Prospective trial of Treat-andExtend versus monthly dosing for neovascular age-related macular degeneration. Ophthalmology 2015;122:2514-22.

35 Wykoff CC, Ou WC, Brown DM, et al. Randomized trial of Treat-andExtend versus monthly dosing for neovascular age-related macular degeneration. Ophthalmology Retina 2017;1:314-21.

36 Silva R, Berta A, Larsen M, et al. Treat-and-Extend versus monthly regimen in neovascular age-related macular degeneration. Ophthalmology 2018;125:57-65.

37 Eichenbaum D, Bann-Mo D, Bhakta A, et al. "It's the Patient Not the Agent: An Assessment of Anti-VEGF Extended Dosing Intervals in Patients with Wet nAMD." Poster on Demand. Chicago: American Society of Retina Specialists, 2019.

38 Stone TW, Hahn P, Raef S, eds. ASRS 2019 Preferences and Trends Membership Survey, 2019.

39 Hussain RM, Hariprasad SM, Ciulla TA. Treatment Burden in Neovascular AMD:Visual Acuity Outcomes are Associated With AntiVEGF Injection Frequency. Ophthalmic Surgery, Lasers and Imaging Retina 2017;48:780-4.

40 Rofagha S, Bhisitkul RB, Boyer DS, et al. Seven-Year outcomes in Ranibizumab-Treated patients in anchor, marina, and horizon. Ophthalmology 2013;120:2292-9.

41 Study of the efficacy and safety of the ranibizumab Port delivery system (RPDS) for sustained delivery of ranibizumab in participants with subfoveal neovascular age-related macular degeneration (AMD) (ladder). Available: https://clinicaltrials.gov/ct2/show/study/ NCT02510794 [Accessed 14 Apr 2019].

42 A study to evaluate the efficacy and safety of KSI-301, an antiVEGF antibody biopolymer conjugate, versus aflibercept in patients with neovascular (wet) age-related macular degeneration. (dazzle). Available: https://clinicaltrials.gov/ct2/show/NCT04049266 [Accessed 9 Sep 2019].

43 A depot formulation of sunitinib malate (GB-102) compared to aflibercept in subjects with wet AMD (ALTISSIMO). Available: https:// clinicaltrials.gov/ct2/show/NCT03953079 [Accessed 9 Sep 2019].

44 Liu K, Song Y, Xu G, et al. Conbercept for treatment of neovascular age-related macular degeneration: results of the randomized phase 3 Phoenix study. Am J Ophthalmol 2019;197:156-67.

45 A study to evaluate the efficacy and safety of Faricimab in participants with neovascular age-related macular degeneration (TENAYA). Available: https://clinicaltrials.gov/ct2/show/ NCT03823287 [Accessed 9 Sep 2019].

46 Study of PAN-90806 eye drops, suspension for neovascular AMD, 2019. Available: https://clinicaltrials.gov/ct2/show/NCT03479372

47 RGX-314 gene therapy for neovascular AMD trial, 2019. Available: https://clinicaltrials.gov/ct2/show/NCT03066258

48 ADVM-022 gene therapy for wet AMD (optic). Available: https:// clinicaltrials.gov/ct2/show/NCT03748784 [Accessed 9 Sep 2019]. 